\title{
INTRODUCCIÓN AL DERECHO INTERNACIONAL PENAL
}

Héctor Olasolo Alonso
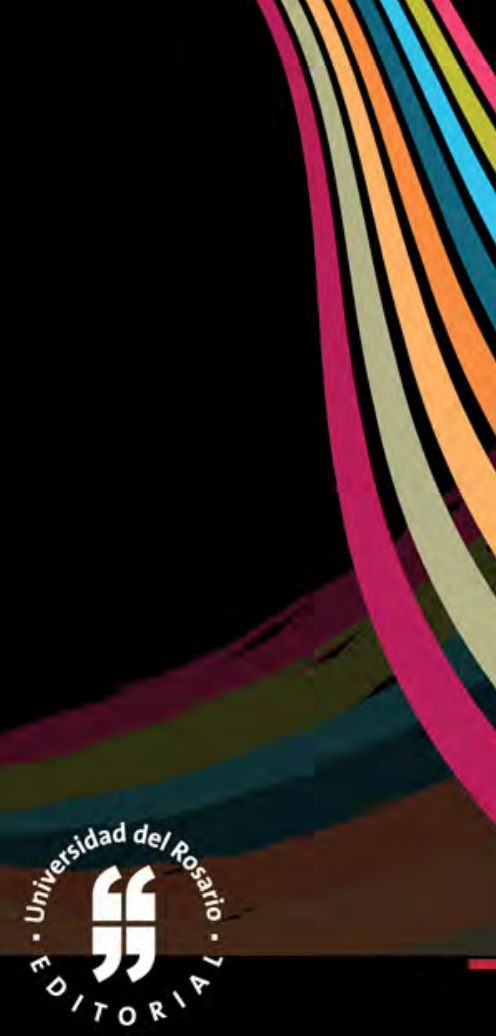

111

11

111

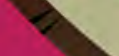




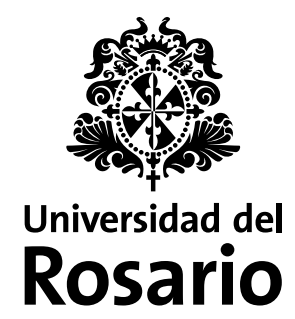



Introducción al derecho internacional penal 


\section{Olasolo Alonso, Héctor}

Introducción al derecho internacional penal / Héctor Olasolo Alonso.— Bogotá: Editorial Universidad del Rosario, Facultad de Jurisprudencia, 2015.

xviii, 220 páginas. - (Colección Textos de Jurisprudencia)

ISBN: 978-958-738-621-9 (rústica)

ISBN:978-958-738-622-6 (digital)

Derecho penal / Derecho internacional / Delitos internacionales / Derecho internacional humanitario / Corte Penal Internacional / Procedimiento penal / I. Título / II. Serie.

341.77

SCDD 20

Catalogación en la fuente - Universidad del Rosario. Biblioteca

amv

Hecho el depósito legal que marca el Decreto 460 de 1995 


\section{Introducción al derecho internacional penal}

Héctor Olasolo Alonso 


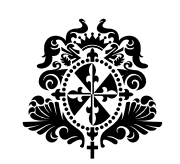

Colección Textos de Jurisprudencia

(C) Editorial Universidad del Rosario

(C) Universidad del Rosario, Facultad de Jurisprudencia

(C) Héctor Olasolo Alonso

Editorial Universidad del Rosario

Carrera 7 No 12B-41, oficina 501 • Teléfono 2970200

editorial.urosario.edu.co
Primera edición: Bogotá D. C., julio de 2015

ISBN: 978-958-738-621-9 (rústica) ISBN: 978-958-738-622-6 (digital)

Coordinación editorial: Editorial Universidad del Rosario Corrección de estilo: Gustavo Patiño Díaz Diseño de cubierta: Kelly Narváez Diagramación: Martha Echeverry Impresión: Xpress. Estudio Gráfico y Digital S.A.

Impreso y hecho en Colombia Printed and made in Colombia

Fecha de evaluación: 30 de septiembre de 2014

Fecha de aceptación: 13 de enero de 2015

Todos los derechos reservados. Esta obra no puede ser reproducida sin el permiso previo por escrito de la Editorial Universidad del Rosario. 


\section{Contenido}

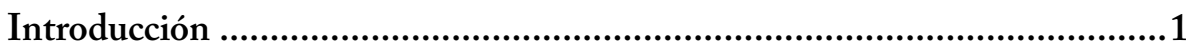

\section{Capítulo 1}

Dignidad humana, derecho internacional penal

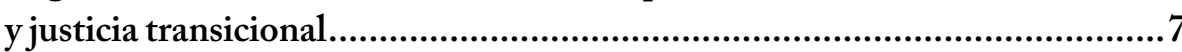

1.1. Introducción: la tradicional subordinación de los recursos

humanos y tecnológicos al conflicto armado

1.2. El reconocimiento de la dignidad humana y el nacimiento

del derecho internacional penal tras la Segunda

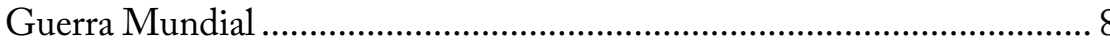

1.3. La inaplicación del derecho internacional penal durante

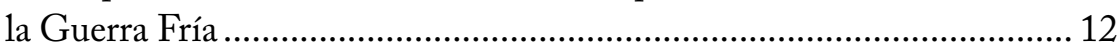

1.4. El resurgimiento de la aplicación del derecho internacional penal al finalizar la Guerra Fría ............................................................. 14

1.5. La aplicación del derecho internacional penal y su incidencia sobre los más recientes procesos de paz

1.6. Conclusión: la aplicación del derecho internacional penal a los máximos responsables como paradigma de validez de las medidas propuestas por la justicia de transición

\section{Capítulo 2}

Los exámenes preliminares de la Corte Penal Internacional en América Latina: el caso colombiano y su impacto sobre futuras negociaciones de paz en la región ....................................................25

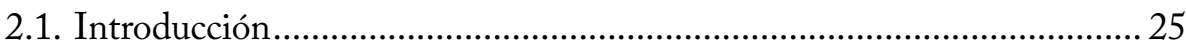

2.2. Los exámenes preliminares de la Fiscalía de la CPI sobre

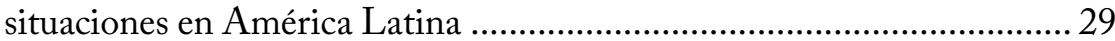

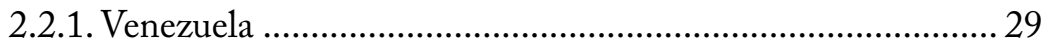

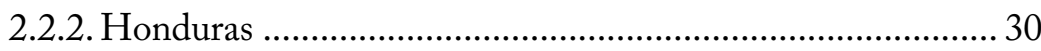




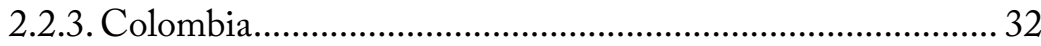

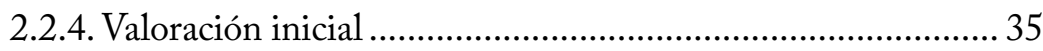

2.3. El fenómeno de la lesa humanidad en Colombia según

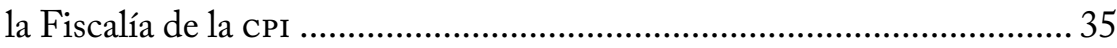

2.4. Consecuencias de la configuración de los delitos de lesa humanidad como crímenes internacionales de ius cogens ......................... 39

2.5. El impacto del examen preliminar de la Fiscalía de la CPI sobre la situación en Colombia en las negociaciones de paz entre el gobierno y las FARC 48

2.6. Conclusión: el impacto del caso colombiano en futuras negociaciones de paz en América Latina ................................................ 55

\section{Capítulo 3}

El principio nullum crimen sine iure en el derecho internacional

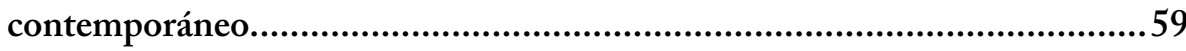

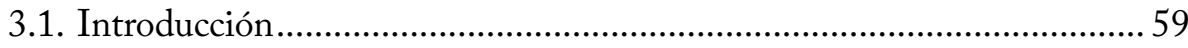

3.2. La evolución del principio nullum crimen sine iure en el ordenamiento jurídico internacional: de "principio de justicia" a "derecho subjetivo individual".

3.3. El contenido del principio nullum crimen sine iure en los sistemas universal y europeo de protección de los derechos humanos 66

3.4. El contenido del principio nullum crimen sine iure en la jurisprudencia de los tribunales internacionales penales

3.5. La extensión del contenido del principio nullum crimen sine iure en el sistema interamericano de protección de los derechos humanos

3.6. La extensión del contenido del principio nullum crimen sine iure en el sistema del ER de la CPI.....

3.7. Exclusión de las cuestiones jurisdiccionales, procesales y procedimentales del ámbito de aplicación del principio nullum crimen sine iure 80

3.8. Conclusión 82 


\section{Capítulo 4}

Las distintas formas de responsabilidad principal

en el artículo 25 (3) (a) del Estatuto de Roma: autoría material, autoría mediata, coautoría por dominio funcional

y coautoría mediata

4.1. Introducción: distinción entre autoría y participación

en el Estatuto de Roma de la Corte Penal Internacional .......................... 85

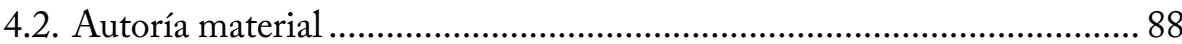

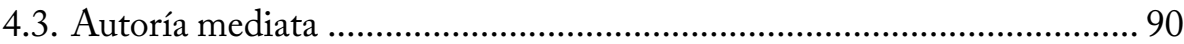

4.3.1. Primera aproximación al concepto …………...................... 90

4.3.2. Autoría mediata a través de personas que no son plenamente responsables...................................................... 93

4.3.3. Autoría mediata a través de personas plenamente responsables: el dominio de la organización.......................... 94

4.4. La coautoría por dominio funcional .................................................... 103

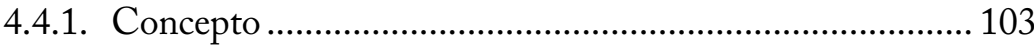

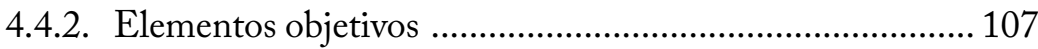

4.4.3. Elementos subjetivos ….................................................. 112

4.5. La cuestión de los superiores intermedios: ¿autoría mediata

por dominio de la organización o coautoría por dominio

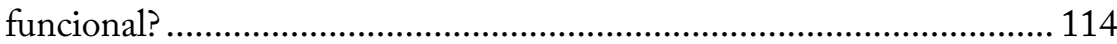

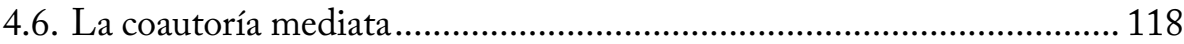

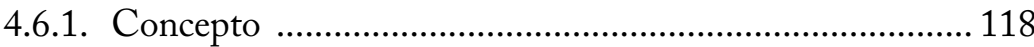

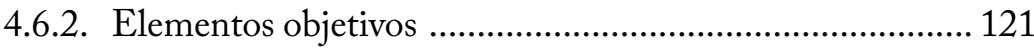

4.6.3. Elementos subjetivos ....................................................... 122

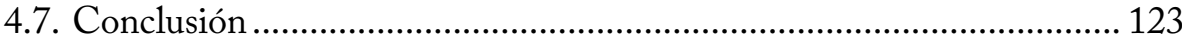

\section{Capítulo 5}

Diálogo jurisprudencial en materia de acceso, participación

y reparación de las víctimas entre el sistema interamericano

de protección de derechos humanos y el sistema de aplicación del derecho internacional penal del Estatuto de Roma...............................125

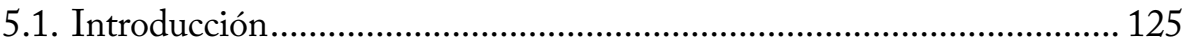

5.2. Primera aproximación a la función de la cidH y de la Corte IDH en el sistema interamericano de derechos humanos ....................... 129

5.3. Definición de víctima según la cidH y la Corte IDH ............................... 132 
5.4. Acceso y participación de las víctimas en el sistema interamericano de protección de derechos humanos

5.4.1. Acceso y participación ante la cidH ................................. 135

5.4.2. Acceso y participación ante la Corte IDH .......................... 144

5.5. Contenido de los derechos fundamentales de las víctimas a la verdad y a la justicia, y de las correspondientes

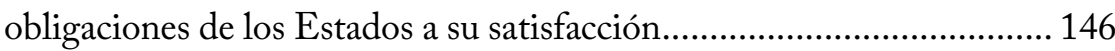

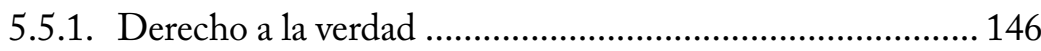

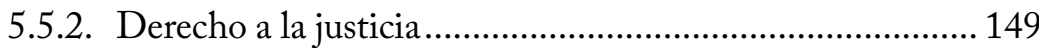

5.6. Reparaciones en el ámbito de la jurisprudencia

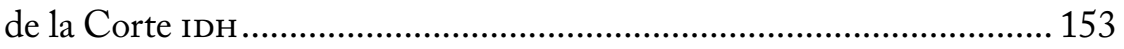

5.7. Primer acercamiento a la posición de la víctima en el ER de la CPI

5.8. Impacto de la jurisprudencia de la Corte IDH en la interpretación de la definición de víctima contenida en la regla 85 de las RPP.

5.9. Impacto de la jurisprudencia de la Corte IDH en relación con el acceso y participación de las víctimas en las actuaciones ante la CPI

5.10. El impacto de la jurisprudencia de la Corte IDH en relación con el contenido del derecho de las víctimas a la reparación, previsto en el artículo 75 del ER

5.11. Conclusión 174

\section{Epílogo}

Propuesta pedagógica de la Clínica Jurídica de Derecho

Internacional Penal y Humanitario de la Universidad del Rosario:

la formación integral del ser humano como un todo

1. Introducción: ¿Es necesaria una autorreflexión sobre el proceso formativo ofrecido por la educación superior?

2. Primera aproximación a la propuesta de la Clínica Jurídica de Derecho Internacional Penal y Humanitario de la Universidad del Rosario

3. La formación integral del pensamiento crítico 181

4. Formación integral del inconsciente: la formación del ser humano como un todo requiere no compartimentar conciencia e inconsciente

5. Conclusión 


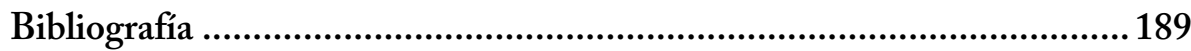

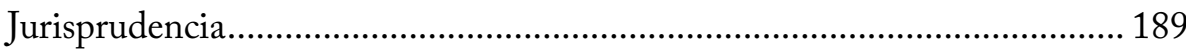

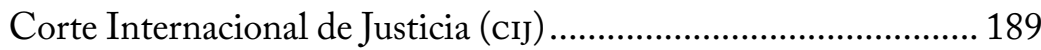

Corte Internacional Permanente de Justicia (cipJ) ....................... 189

Tribunal Internacional Militar-Núremberg (тім) ........................ 189

Tribunal Internacional Militar para el Lejano

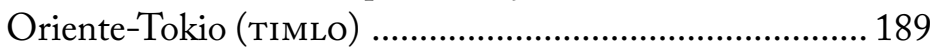

Corte Europea de Derechos Humanos (стEDH).......................... 189

Comisión Interamericana de Derechos Humanos (CIDH) ............ 190

Corte Interamericana de Derechos Humanos (Corte IDH)........... 190

Corte Penal Internacional (cPI)................................................. 194

Tribunal Internacional Penal para la ex Yugoslavia (TIPY)............ 198

Tribunal Internacional Penal para Ruanda (TIPR) ......................... 199

Corte Especial para Sierra Leona (CESL)...................................... 199

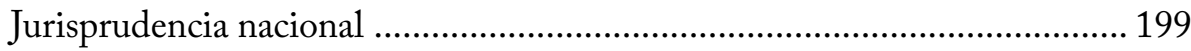

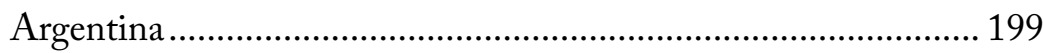

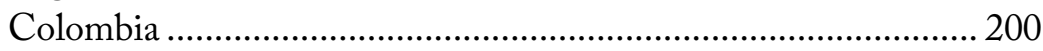

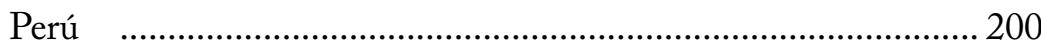



A Aida Cecilia Gómez Solano, Valentín Olasolo Castéllet y Mercedes Alonso Cañas, por su apoyo continuo durante la redacción del presente trabajo. A Carsten Stahn, por su hospitalidad durante mis estancias en La Haya. A la Facultad de Jurisprudencia de la Universidad del Rosario y a su Área de Derecho Internacional, por la cálida acogida con la que me han dispensado. 



\section{Lista de siglas y abreviaturas}

$\begin{array}{ll}\text { AECID } & \text { Agencia Española de Cooperación Internacional para el } \\ & \text { Desarrollo } \\ \text { ART(s). } & \text { artículo(s) } \\ \text { AUC } & \text { Autodefensas Unidas de Colombia } \\ \text { CDI } & \text { Comisión de Derecho Internacional } \\ \text { CADH } & \text { Convención Americana de Derechos Humanos } \\ \text { CDH } & \text { Comité de Derechos Humanos } \\ \text { CEDDHH } & \text { Comisión Europea de Derechos Humanos } \\ \text { CEDH } & \text { Convención Europea de Derechos Humanos } \\ \text { CELS } & \text { Centros de Estudios Legales y Sociales } \\ \text { CESL } & \text { Corte Especial para Sierra Leona } \\ \text { CG I, II, III y IV } & \text { Convenciones de Ginebra I, II, III y IV de 1949 } \\ \text { CICIG } & \text { Comisión Internacional contra la Impunidad en Guatemala } \\ \text { CIDH } & \text { Comisión Interamericana de Derechos Humanos } \\ \text { CIDFP } & \text { Convención Interamericana sobre Desapariciones Forzadas } \\ & \text { de Personas } \\ \text { CIPSEVM } & \text { Convención Interamericana para Prevenir, Sancionar y Erra- } \\ & \text { dicar la Violencia contra la Mujer } \\ \text { CIPST } & \text { Convención Interamericana para Prevenir y Sancionar la } \\ & \text { Tortura } \\ \text { COord(s) } & \text { coordinador(es) } \\ \text { Corte IDH } & \text { Corte Interamericana de Derechos Humanos } \\ \text { CPI } & \text { Corte Penal Internacional } \\ \text { CPP } & \text { Comité para la Protección de los Periodistas } \\ \text { CTEDH } & \text { Corte Europea de Derechos Humanos } \\ \text { CVR } & \text { Comisión para la Verdad y la Reconciliación } \\ \text { DADDH } & \text { Declaración Americana sobre Derechos y Deberes del } \\ \text { DINA } & \text { Hombre } \\ & \text { Dirección de Inteligencia Nacional de Chile } \\ & \end{array}$




$\begin{array}{ll}\text { DUDH } & \text { Declaración Universal de los Derechos Humanos } \\ \text { EC } & \text { Elementos de los Crímenes de la Corte Penal Internacional } \\ \text { ECC } & \text { Empresa Criminal Común } \\ \text { ECCC } & \text { Extraordinary Chambers of the Courts of Cambodia (Salas } \\ & \text { Extraordinarias de las Cortes de Camboya) } \\ \text { ECESL } & \text { Estatuto de la Corte Especial para Sierra Leona } \\ \text { ECIDH } & \text { Estatuto de la Comisión Interamericana de Derechos } \\ & \text { Humanos } \\ \text { ECIJ } & \text { Estatuto de la Corte Internacional de Justicia } \\ \text { ED. } & \text { edición } \\ \text { EE. UU. } & \text { Estados Unidos de América } \\ \text { ELN } & \text { Ejército de Liberación Nacional } \\ \text { ESETC } & \text { Estatuto de las Salas Extraordinarias de los Tribunales de } \\ & \text { Camboya } \\ \text { ETEL } & \text { Estatuto del Tribunal Especial para el Líbano } \\ \text { ETIM } & \text { Estatuto del Tribunal Internacional Militar/Tribunal de } \\ & \text { Núremberg } \\ \text { ETIMLO } & \text { Estatuto del Tribunal Internacional Militar para el Lejano } \\ & \text { Oriente/Tribunal de Tokio } \\ \text { ETIPR } & \text { Estatuto del Tribunal Internacional Penal para Ruanda } \\ \text { ETIPY } & \text { Estatuto del Tribunal Internacional Penal para la ex-Yugoslavia } \\ \text { ER } & \text { Estatuto de Roma de la Corte Penal Internacional } \\ \text { FARC } & \text { Fuerzas Armadas Revolucionarias de Colombia } \\ \text { FNI } & \text { Frente Nacionalista e Integracionista } \\ \text { FPLC } & \text { Fuerzas Patrióticas para la Liberación de Congo } \\ \text { FRPI } & \text { Fuerzas de Resistencia Patriótica de Ituri } \\ \text { idem } & \text { misma obra y misma página } \\ \text { ibid. } & \text { misma obra } \\ \text { VRS/JNA } & \text { Ejército Serbo-Bosnio } \\ \text { MLC } & \text { Movimiento por la Liberación de Congo } \\ \text { MJE } & \text { Movimiento por la Justicia y la Equidad } \\ \text { MONUC } & \text { Misión de las Naciones Unidas en Congo } \\ \text { MSL } & \text { Movimiento Sudanés por la Liberación } \\ \text { NÚM(s). } & \text { número(s) } \\ \text { ONG } & \text { Organización de Estados Americanos } \\ & \text { organización no gubernamental } \\ & \end{array}$




\begin{tabular}{|c|c|}
\hline $\begin{array}{l}\text { ONU } \\
\text { op. cit. }\end{array}$ & $\begin{array}{l}\text { Organización de las Naciones Unidas } \\
\text { obra citada }\end{array}$ \\
\hline OTAN & Organización del Tratado del Atlántico Norte \\
\hline PA I & $\begin{array}{l}\text { Protocolo Adicional i a las Convenciones de Ginebra de 1949, } \\
\text { Aplicable a los Conflictos Armados de Carácter Internacional }\end{array}$ \\
\hline A II & $\begin{array}{l}\text { Protocolo Adicional in a las Convenciones de Ginebra de } \\
\text { 1949, Aplicable a los conflictos Armados de Carácter no } \\
\text { Internacional }\end{array}$ \\
\hline ACADHDESC & $\begin{array}{l}\text { Protocolo Adicional a la Convención Americana sobre De- } \\
\text { rechos Humanos en Materia de Derechos Económicos, So- } \\
\text { ciales y Culturales }\end{array}$ \\
\hline o. & página \\
\hline pp. & páginas \\
\hline PÁRR(s). & párrafo(s) \\
\hline PCADHAPM & $\begin{array}{l}\text { Protocolo a la Convención Americana sobre Derechos Hu- } \\
\text { manos Relativos a la Abolición de la Pena de Muerte }\end{array}$ \\
\hline IIDCP & Pacto Internacional de Derechos Civiles y Políticos \\
\hline RAK & República Autónoma de Krajina \\
\hline $\mathrm{RCA}$ & República Centro-Africana \\
\hline $\mathrm{R}$ CIDH & $\begin{array}{l}\text { Reglamento de la Convención Interamericana de Derechos } \\
\text { Humanos }\end{array}$ \\
\hline RCorteIDH & Reglamento de la Corte Interamericana de Derechos Humanos \\
\hline REGC & Reglamento de la Corte Penal Internacional \\
\hline $\mathrm{RDC}$ & República Democrática del Congo \\
\hline RPP & $\begin{array}{l}\text { Reglas de Procedimiento y Prueba de la Corte Penal } \\
\text { Internacional }\end{array}$ \\
\hline RTS & Radio Televisión Serbia \\
\hline SCP I, II Y III & $\begin{array}{l}\text { Sala de Cuestiones Preliminares I, i y iII de la Corte Penal } \\
\text { Internacional }\end{array}$ \\
\hline SETC & Salas Extraordinarias de los Tribunales de Camboya \\
\hline $\sin$ & Servicio de Inteligencia Nacional del Perú \\
\hline SPI & Sala de Primera Instancia \\
\hline SA & Sala de Apelaciones \\
\hline ss. & Siguientes \\
\hline TEL & Tribunal Especial para el Líbano \\
\hline TIM & Tribunal Internacional Militar/Tribunal de Núremberg \\
\hline
\end{tabular}




$\begin{array}{ll}\text { TIMLO } & \begin{array}{l}\text { Tribunal Internacional Militar para el Lejano Oriente/Tri- } \\ \text { bunal de Tokio }\end{array} \\ \text { TIPR } & \text { Tribunal Internacional Penal para Ruanda } \\ \text { TIPY } & \text { Tribunal Internacional Penal para la ex-Yugoslavia } \\ \text { UNMIK } & \text { Misión de las Naciones Unidas para Kósovo } \\ \text { UNTAET } & \text { Misión de las Naciones Unidas para Timor Oriental } \\ \text { UPC/RP } & \text { Unión Patriótica Congolesa } \\ \text { UPDF } & \text { Ugandan People Defence Forces (Fuerzas de Defensa Popular } \\ & \text { de Uganda) } \\ \text { vid. } & \text { véase } \\ \text { vol(s). } & \text { volumen(es) }\end{array}$




\section{Introducción}

La presente obra recoge una colección de ensayos escritos desde mi llegada a la Universidad del Rosario, en Bogotá, en febrero de 2013. En ellos se aborda un conjunto de principios que considero fundamentales para la comprensión y aplicación del derecho internacional penal. El título elegido, Introducción al derecho internacional penal, pretende reflejar esta realidad.

El lector encontrará varias novedades frente a trabajos anteriores. En primer lugar, desde una perspectiva estrictamente formal, observará que la tilde de Olasolo ha sido eliminada para que el apellido vuelva a su estado natural,y revertir así la castellanización que con dicha tilde se pretendió realizar durante los tiempos de la dictadura de Francisco Franco.

En segundo lugar, el lector encontrará un giro hacia un análisis más conceptual e interdisciplinario del fundamento y función del derecho internacional penal en la sociedad global. Este giro aparece desde el propio título del trabajo, que refleja cómo, a diferencia de trabajos anteriores, el énfasis del presente estudio se encuentra en la cuestión relativa al recurso por la sociedad internacional a un concepto jurídico que hasta bien entrado el siglo xx se encontraba exclusivamente en la esfera de los ordenamientos jurídicos nacionales: la responsabilidad penal en que incurre la persona humana al llevar a cabo ciertas omisiones o actos ilícitos.

Con los dos primeros ensayos del presente trabajo se inicia el camino hacia una mayor comprensión de las circunstancias históricas, las causas económicas, sociales, políticas, militares y filosóficas, y las finalidades que llevaron a la sociedad internacional a recurrir a dicho concepto jurídico, así como de la evaluación que las mismas han sufrido durante los más de 40 años de Guerra Fría y de 25 que han transcurrido desde la finalización de esta.

Es necesario entender que la configuración de la persona humana como sujeto de obligaciones en el ordenamiento jurídico internacional (cuyo incumplimiento le hace incurrir en responsabilidad penal), se produce simultáneamente con su reconocimiento como sujeto de derechos a través del derecho 
internacional de los derechos humanos. La interrelación entre los derechos a la verdad, la justicia y la reparación de las víctimas de crímenes internacionales, y la responsabilidad penal internacional de quienes los cometen, adquiere de esta manera una relevancia fundamental.

La comprensión de todas estas circunstancias y del funcionamiento de los mecanismos centralizados (tribunales internacionales penales) y descentralizados (jurisdicciones nacionales de los Estados directamente afectados o que actúan con base en el principio de justicia universal) de los que la sociedad internacional se ha dotado para la declaración y realización de la responsabilidad internacional penal permite ir más allá del análisis de las cuestiones de técnica jurídica (características de la gran mayoría de los trabajos que hasta ahora se vienen produciendo), para centrarnos en el desarrollo de una política criminal internacional que aborde aquellos fenómenos que constituyen una auténtica amenaza para la existencia material de la propia sociedad internacional. La presente obra constituye un primer paso en esta dirección.

En cuanto al contenido específico de los ensayos presentados en este volumen, el primero analiza el contexto histórico al finalizar la is Guerra Mundial: el temor a un nuevo conflicto armado de dimensiones globales capaz de destruir definitivamente el planeta y que exigía un cambio significativo en la praxis internacional. Fue este contexto particular el que propició la afirmación del paradigma sobre el que se construye el derecho internacional penal: quienes desde los resortes del poder recurren a una guerra de agresión contra terceros Estados y utilizan la fuerza armada contra su propia población, no solo pierden la legitimidad ética y moral necesaria para seguir dirigiendo sus respectivas sociedades, sino que, debido al daño que han causado a la sociedad internacional, incurren jurídicamente frente a la misma en responsabilidad penal individual, que no se extingue bajo ninguna circunstancia.

Estudiadas las razones que evitaron, en gran medida, su aplicación durante la Guerra Fría, así como aquellas otras que lo han reafirmado en los últimos 25 años, el ensayo constata la resistencia al cambio de aproximación que esto requiere frente a los procesos de paz. Esta exigencia resulta inevitable si se tiene en cuenta que antes del advenimiento de los tribunales internacionales penales a mediados de los noventa, los procesos de paz se caracterizaban por que los dirigentes de un pasado de violencia, muerte y destrucción recibían el respaldo de la geoestrategia internacional para continuar liderando sus 
sociedades hacia supuestos futuros de paz, que, como regla general, nunca llegaban a consolidarse.

El segundo ensayo entronca de manera lógica con el primero al desarrollar el contenido y naturaleza normativa del principio "no hay paz sin justicia", que requiere a los dirigentes dejar inmediatamente sus posiciones de liderazgo y hacer frente a la responsabilidad penal asumida frente a su propia sociedad y a la sociedad internacional en su conjunto. El ensayo aplica las implicaciones de esta exigencia al caso concreto de las negociaciones de paz actualmente desarrolladas entre el gobierno de Colombia y las Fuerzas Armadas Revolucionarias de Colombia, a la luz del examen preliminar realizado desde 2005 por la Fiscalía de la Corte Penal Internacional (CPI).

El tercer ensayo estudia el contenido del que entendemos que constituye el principio central de la parte general del derecho internacional penal: nullum crimen sine iure. Se describe en primer lugar el paso de su configuración inicial como principio de justicia a su actual expresión como derecho subjetivo individual, cuyo ámbito de aplicación no incluye las cuestiones jurisdiccionales, procesales y procedimentales. A continuación se analiza su contenido en los sistemas universal, europeo e interamericano de protección de derechos humanos, así como en la jurisprudencia de los tribunales internacionales penales y la CPI, para concluir que el derecho internacional general no exige que el derecho aplicable provenga de una lex scripta ni recoge garantías de certeza en cuanto a qué comportamientos son constitutivos de delito, más allá de los requisitos de accesibilidad y previsibilidad. El ensayo termina subrayando que toda configuración, por vía interpretativa, de un contenido reforzado del principio nullum crimen sine iure (tal como la Corte IDH y la CPI han llevado a cabo) debe ser compatible con el resto de obligaciones impuestas a los Estados por los sistemas de protección universal y regional de derechos humanos, y en particular con sus deberes de investigar y enjuiciar a los autores de graves violaciones de derechos humanos y de garantizar a las víctimas el pleno disfrute de su derecho a un recurso judicial efectivo para la investigación y enjuiciamiento de los presuntos responsables.

El cuarto ensayo desarrolla las diferentes manifestaciones del concepto de autor en el derecho internacional penal, con especial atención al artículo 25 (3) (a) del Estatuto de Roma (ER). El ensayo analiza las categorías de autoría material, autoría mediata, coautoría y coautoría mediata, con miras a 
reforzar la función preventiva del derecho internacional penal, mediante la intervención sobre los centros de poder donde se adoptan las decisiones acerca del diseño y ejecución de campañas de violencia sistemática y/o a gran escala constitutivas de genocidio, delitos de lesa humanidad y crímenes de guerra.

El quinto ensayo aborda la cuestión del diálogo jurisprudencial entre los tribunales internacionales que tienen a su cargo la aplicación del derecho internacional penal y el derecho internacional de los derechos humanos. Se estudia en particular el notable impacto de las decisiones de la Corte IDH en ciertos ámbitos de la jurisprudencia de la CPI, tales como el acceso de las víctimas a la CPI con base en sus derechos a la verdad y la justicia, la configuración de la categoría de víctimas indirectas y las diversas modalidades de reparación.

La obra finaliza con un acercamiento a las actividades pedagógicas en el ámbito del derecho internacional penal, mediante el análisis del proyecto de formación integral de la Clínica Jurídica de Derecho Internacional Penal y Humanitario de la Universidad del Rosario, que realiza actividades de asesoría jurídica pro bono para la Oficina de Víctimas de la cPI y la jurisdicción de justicia y paz en Colombia.

Conforme a esta propuesta, el estudiante pasa a ser la figura central en el proceso de formación, tomando conciencia de que el conocimiento se obtiene principalmente por medio de la observación, la experimentación y la combinación de razonamientos. En este proceso de naturaleza no directiva, la función principal del profesor es suscitar problemas y plantear diversas alternativas posibles a los mismos, que tendrán que ser resueltos mediante el trabajo en equipo. Al mismo tiempo, la dimensión subjetiva o inconsciente del estudiante es potenciada para que este último pueda articular una forma coherente de ser y de entender el mundo, mediante la discusión sobre los valores y los problemas humanos y sociales, y el fomento del sueño de los jóvenes por una sociedad internacional distinta que haga que la vida tenga un sentido para ellos.

Antes de terminar esta introducción, quiero agradecer a la Editorial Universidad del Rosario y a la Editorial Tirant lo Blanch por la confianza que han depositado en mí desde hace ya un buen número de años, y que con el presente proyecto no hace sino renovarse una vez más.

Quiero también agradecer al lector por su interés en la obra y su crítica de la misma, la cual, como afirma Walter Benjamin —el gran filósofo judíoalemán de la Escuela de Frankfurt que se suicidó en Portbou en septiembre 
de 1940, mientras trataba de cruzar por los Pirineos la frontera española en su huida de la persecución del régimen nazi-, no hace sino completar en el momento presente el trabajo inicialmente realizado por el autor.

Bogotá, 23 de julio de 2014 